\title{
Permutation Reconstruction from Minors
}

\author{
Mariana Raykova \\ Bard College \\ Annandale-on-Hudson, NY 12504, USA \\ mr892@bard.edu
}

Submitted: Aug 19, 2005; Accepted: Jul 29, 2006; Published: Aug 3, 2006

Mathematics Subject Classification: 05A05

\begin{abstract}
We consider the problem of permutation reconstruction, which is a variant of graph reconstruction. Given a permutation $p$ of length $n$, we delete $k$ of its entries in each possible way to obtain $\left(\begin{array}{l}n \\ k\end{array}\right)$ subsequences. We renumber the sequences from 1 to $n-k$ preserving the relative size of the elements to form $(n-k)$-minors. These minors form a multiset $M_{k}(p)$ with an underlying set $M_{k}^{\prime}(p)$. We study the question of when we can reconstruct $p$ from its multiset or its set of minors. We prove there exists an $N_{k}$ for every $k$ such that any permutation with length at least $N_{k}$ is reconstructible from its multiset of $(n-k)$-minors. We find the bounds $N_{k}>k+\log _{2} k$ and $N_{k}<\frac{k^{2}}{4}+2 k+4$. For the number $N_{k}^{\prime}$, giving the minimal length for permutations to be reconstructible from their sets of $(n-k)$-minors, we have the bound $N_{k}^{\prime}>2 k$. We work towards analogous bounds in the case when we restrict ourselves to layered permutations.
\end{abstract}

\section{Introduction}

The problem of graph reconstruction arose from an unsolved conjecture of Ulam [5]. Consider any two unlabeled simple graphs $A$ and $B$ each with $n>3$ vertices. Deleting one vertex from $A$ together with its incident edges in each possible way we obtain the minors $A_{1}, \ldots, A_{n}$. Similarly, obtain minors $B_{1}, \ldots, B_{n}$ of $B$. Then, Ulam's conjecture says that if there exists a bijection $\alpha:\{1, \ldots, n\} \rightarrow\{1, \ldots, n\}$ such that $A_{i}$ is isomorphic to $B_{\alpha(i)}$, then $A$ is isomorphic to $B$.

We consider a variation of graph reconstruction introduced by Smith [4]. We apply the construction from the approach in graph reconstruction to permutations. Consider a permutation $p$ with $n$ entries. Delete $k$ of the entries in each possible way to obtain $\left(\begin{array}{l}n \\ k\end{array}\right)$ subsequences of the starting permutation and then renumber them with respect to order so that they become permutations of the numbers from 1 to $n-k$. These resulting subpermutations are called $(n-k)$-minors and the multiset of these minors is denoted by $M_{k}(p)$. We ask when the multiset $M_{k}(p)$ determines the permutation $p$. Smith [4] introduced 
the problem of permutation reconstruction and looked at the number $N_{k}$, defined to be the smallest number such that we can reconstruct permutations of length $n \geq N_{k}$ from their multisets of $(n-k)$-minors. She found the values $N_{1}=5, N_{2}=6$ and gave an upper bound $N_{3} \leq 13$. She also stated a conjecture that $N_{k}=k+4$, but she did not prove the existence of $N_{k}$ for every positive integer $k$.

We consider the problem of how long a permutation $p$ should be so that it can be reconstructed from its multiset of $(n-k)$-minors $M_{k}(p)$, or from the underlying set $M_{k}^{\prime}(p)$, which in most cases is different from $M_{k}(p)$.

In Section 2 of the paper we prove that $N_{k}$ exists for all values of $k$. In Section 3 we give an upper bound $N_{k}<\frac{k^{2}}{4}+2 k+4$. We also obtain a lower bound $N_{k}>k+\log _{2} k$ in Section 4 that disproves Smith's conjecture [3].

In Section 5 we consider the set $M_{k}^{\prime}(p)$ and the corresponding number $N_{k}^{\prime}$ giving the minimal length for permutations to be reconstructible from their sets of minors. We prove that if permutations of length $n$ are reconstructible from the sets of their $(n-k)$-minors, the same is true for permutations of greater lengths. We give a lower bound $N_{k}^{\prime}>2 k$ together with the exact values $N_{1}^{\prime}=5$ and $N_{2}^{\prime}=7$. We use some of the results on permutation reconstruction from the sets of $(n-k)$-minors to determine the exact value $N_{3}=7$.

We do not know whether $N_{k}^{\prime}<\infty$. One approach to prove this is to start considering specific types of permutations and try to answer the question for them. In Section 6 we study reconstruction of a certain type of pattern avoiding permutations, layered permutations. Section 7 states some open questions for further work.

\section{Reconstruction from Multisets}

In this section we prove the existence of $N_{k}$ for every positive integer $k$ and give the exact values for $N_{1}$ and $N_{2}$.

Definition 2.1. Let $p$ be a permutation of length $n$. An $(n-k)$-minor of $p$ is a length $n-k$ subsequence of $p$ with entries renumbered $1,2, \ldots, n-k$ preserving order. Let $M_{k}(p)$ denote the multiset of all $(n-k)$-minors of $p$.

We obtain an $(n-k)$-minor of a permutation $p$ of length $n$ by deleting $k$ of its entries and then renumbering the remaining subsequence in such a way that the entry at position $i$ will be greater than the entry at position $j$ in the resulting permutation if and only if the entry at position $i$ was greater than the entry at position $j$ in the starting subsequence. We use the notation $M_{k}(p)=\left\{q_{1}^{k_{1}}, \ldots, q_{r}^{k_{r}}\right\}$ to denote $k_{1}$ copies of $q_{1}, \ldots, k_{r}$ copies of $q_{r}$ in the multiset of $(n-k)$-minors of $p$.

Example 2.2. Consider the permutation $p=51432$. When we delete two entries of $p$ in each possible way, we get the following subsequences:

$$
\{514,513,512,543,542,532,143,142,132,432\} \text {. }
$$

After renumbering them we end up with the multiset of (3)-minors of $p$ :

$$
M_{2}(51432)=\left\{132^{3}, 312^{3}, 321^{4}\right\}
$$


Definition 2.3. Let $N_{k}$ be the smallest integer such that for all permutations $p$ and $q$ of length $n \geq N_{k}, M_{k}(p)=M_{k}(q)$ implies $p=q$. If no such integer exists we will write $N_{k}=\infty$.

If the number $N_{k}$ exists for some $k$, then distinct permutations of length $n \geq N_{k}$ have distinct multisets of $(n-k)$-minors and there exist distinct permutations $q$ and $r$ of length $N_{k}-1$ such that $M_{k}(q)=M_{k}(r)$.

Notation 2.4. Let $p$ be a permutation of length $n$. For any $1 \leq i, j \leq n$, we will say $i<_{p} j$ if $i$ is to the left of $j$ in $p$ and $i>_{p} j$ if $i$ is to the right of $j$ in $p$.

Notation 2.5. We denote the entry at position $i$ in the permutation $p$ by $p(i)$.

We now proceed with the proof of the main theorem for the section.

Theorem 2.6. For every positive integer $k$ the number $N_{k}$ exists.

Proof. For all $2 \leq i \leq k+1$ consider the inequalities:

$$
n^{2}-n[2 k+1+i(k-i+2)]+(k+1)[k+(i-1)(k-i+2)]>0
$$

Since the inequalities 2.1 are of the form $n^{2}-O(n)$, there exists a number $N$ such that the inequalities are satisfied for all $n \geq N$.

Let $p$ be a permutation of length $n \geq N$ with a multiset of $(n-k)$-minors $M_{k}(p)$.

We will give an algorithm for reconstructing $p$ from the multiset $M_{k}(p)$ by determining the relative positions of the entries of $p$.

First we will determine the relative positions of $1,2, \ldots, k+1$ in the permutation $p$. Let $x_{1,2}$ be the number of $(n-k)$-minors $q \in M_{k}(p)$ such that $1<_{q} 2$ and $x_{2,1}$ be the number of $(n-k)$-minors $r \in M_{k}(p)$ such that $2<_{r} 1$.

We now determine the relative positions of 1 and 2 in $p$. We have that $n$ satisfies the inequality obtained from $(2.1)$ for $i=2$ :

$$
n^{2}-n(4 k+1)+2 k(k+1)>0,
$$

which is equivalent to

$$
\left(\begin{array}{c}
n-2 \\
k
\end{array}\right)>\frac{1}{2}\left(\begin{array}{l}
n \\
k
\end{array}\right) .
$$

$M_{k}(p)$ contains $\left(\begin{array}{c}n-2 \\ k\end{array}\right)(n-k)$-minors obtained when neither 1 nor 2 is deleted from $p$. Therefore if $q$ is any of these $(n-k)$-minors, we will have that $1<_{q} 2$ if and only if $1<_{p} 2$. Since $\left|M_{k}(p)\right|=\left(\begin{array}{l}n \\ k\end{array}\right)$, we will have that in more than half of the $(n-k)$-minors of $p 1$ and 2 have the same relative positions as in $p$. Hence if $x_{1,2}>x_{2,1}$ then $1<_{p} 2$, and if $x_{1,2}<x_{2,1}$ then $1>_{p} 2$.

Now we will assume that we have determined the relative positions of $1, \ldots, i-1$ in $p$ where $3 \leq i \leq k+1$ and we will determine the relative position of $i$ with respect to $1, \ldots, i-1$. Let $R_{i}=\left\{q \mid q \in M_{k}(p)\right.$ and $q$ is obtained by deleting at most $i-3$ entries from $1, \ldots, i$ in $p\}$. Let $y_{1,2}$ be the number of $(n-k)$-minors $q \in R_{i}$ such that $1<_{q} 2$. Note that $y_{1,2}$ is determined by the relative positions of $1,2, \ldots, i-1$ in $p$. Let $x=x_{1,2}-y_{1,2}$. 
We consider the sets

$$
\begin{gathered}
A=\left\{q \mid q \in M_{k}(p) \text { and } q \text { is obtained from } p\right. \text { by deleting } \\
i-2 \text { entries from } 1, \ldots, i-1 \text { and not deleting } i\} \\
B=\left\{q \mid q \in M_{k}(p) \text { and } q \text { is obtained from } p\right. \text { by deleting } \\
\quad i-2 \text { entries from } 1, \ldots, i-1 \text { and } i\}, \\
C=\left\{q \mid q \in M_{k}(p) \text { and } q \text { is obtained from } p \text { by deleting all entries } 1, \ldots, i-1\right\} .
\end{gathered}
$$

We have that $M_{k}(p)=R_{i} \cup A \cup B \cup C$. Also $|A|=(i-1)\left(\begin{array}{c}n-i \\ k-i+2\end{array}\right),|B|=(i-1)\left(\begin{array}{c}n-i \\ k-i+1\end{array}\right)$ and $|C|=\left(\begin{array}{l}n-i+1 \\ k-i+1\end{array}\right)$. Let $j$ be the number of entries from $\{1,2, \cdots, i-1\}$ that appear before $i$ in $p$. Then we have that there are $j\left(\begin{array}{c}n-i \\ k-i+2\end{array}\right)(n-k)$-minors $q \in A$ such that $1<_{q} 2$. In the $(n-k)$-minors from the sets $B$ and $C$ the relative positions of 1 and 2 are not determined by the relative order of $1, \cdots, i-1, i$ in $p$. Nevertheless, we still know that $j\left(\begin{array}{c}n-i \\ k-i+2\end{array}\right) \leq x \leq j\left(\begin{array}{c}n-i \\ k-i+2\end{array}\right)+|B|+|C|$, which implies

$$
j\left(\begin{array}{c}
n-i \\
k-i+2
\end{array}\right) \leq x \leq j\left(\begin{array}{c}
n-i \\
k-i+2
\end{array}\right)+(i-1)\left(\begin{array}{c}
n-i \\
k-i+1
\end{array}\right)+\left(\begin{array}{c}
n-i+1 \\
k-i+1
\end{array}\right)
$$

From Equation (2.2), we get

$$
\frac{x}{\left(\begin{array}{c}
n-i \\
k-i+2
\end{array}\right)}-\frac{(i-1)\left(\begin{array}{c}
n-i \\
k-i+1
\end{array}\right)+\left(\begin{array}{c}
n-i+1 \\
k-i+1
\end{array}\right)}{\left(\begin{array}{c}
n-i \\
k-i+2
\end{array}\right)} \leq j \leq \frac{x}{\left(\begin{array}{c}
n-i \\
k-i+2
\end{array}\right)} .
$$

However, we assumed that $n$ satisfies the inequality

$$
n^{2}-n[2 k+1+i(k-i+2)]+(k+1)[k+(i-1)(k-i+2)]>0,
$$

which implies

$$
\frac{(i-1)\left(\begin{array}{c}
n-i \\
k-i+1
\end{array}\right)+\left(\begin{array}{c}
n-i+1 \\
k-i+1
\end{array}\right)}{\left(\begin{array}{c}
n-i \\
k-i+2
\end{array}\right)}<1 .
$$

Therefore since $j$ is an integer, it is uniquely determined by (2.3) and we have determined the position of $i$ relative to $1, \ldots, i-1$ in $p$.

So far we have determined the relative positions of $1, \ldots, k+1$ in $p$ and we now determine the relative positions of $k+2, \ldots, n$. Suppose that we have determined the relative order of $1,2, \ldots, j-1$ in $p$ for some $j \geq k+2$. We will show how to determine the position of $j$ relative to $1,2, \ldots, j-1$.

First we will find where $j$ is relative to $1,2, \ldots, k+1$ in $p$. Consider the relative order of 1 and $j-k$ in the $(n-k)$-minors. Let $x_{1, j-k}$ be the number of $(n-k)$-minors $q$ such that $1<_{q} j-k$. Let $R_{j}=\{q \mid q$ is obtained by deleting at most $k-1$ entries from $1,2, \ldots, j-1\}$. Let $y_{1, j-k}$ be the number of $(n-k)$-minors $q \in R_{j}$ such that $1<_{q} j-k$ and let $y=x_{1, j-k}-y_{1, j-k}$. Note that $y_{1, j-k}$ is determined by the relative order of $1, \ldots, j-1$ 
in $p$. Therefore $y$ is the number of $(n-k)$-minors $q$ such that $1<_{q} j-k$ and the entry $j$ in $p$ acts as $j-k$ in $q$.

Now let $r$ be the permutation of $1,2, \ldots, j$ in which the entries have the same relative order as the corresponding entries in $p$. Suppose $r(l)=j$. Then we have that

$$
y=\sum_{m=1}^{l-1}\left(\begin{array}{l}
j-r(m)-1 \\
k-r(m)+1
\end{array}\right)
$$

since there are $\left(\begin{array}{c}j-r(m)-1 \\ k-r(m)+1\end{array}\right)(n-k)$-minors in which the relative order of 1 and $j-k$ is determined by the relative order of $r(m)$ and $j$ in $p$. Since we know the value of $y$ and $\sum_{m=1}^{l_{1}-1}\left(\begin{array}{c}j-r(m)-1 \\ k-r(m)+1\end{array}\right)<\sum_{m=1}^{l_{2}-1}\left(\begin{array}{l}j-r(m)-1 \\ k-r(m)+1\end{array}\right)$ for $l_{1}<l_{2}$, we can conclude that $l$ is uniquely determined by $y$. Hence we can determine the position of $j$ relative to $1,2, \ldots, k+1$ in $p$.

Now suppose that we know the position of $j$ relative to $1,2, \ldots, s-1$ where $k+1<$ $s<j-1$. We will show how to determine the relative order of $j$ and $s$. Consider the relative order of $s-k$ and $j-k$ in the $(n-k)$-minors of $p$. Let $x_{(s-k)(j-k)}$ be the number of $(n-k)$-minors $q$ such that $s-k<_{q} j-k$. Let $R_{s}=\{q \mid q$ is obtained by deleting at most $k-1$ entries from $1, \ldots, s-1\}$. Let $y_{s-k, j-k}$ be the number of $(n-k)$-minors $q \in R_{s}$ such that $s-k<_{q} j-k$. Note that $y_{s-k, j-k}$ is determined by the relative order of $1, \ldots, j-1$ as well as the position of $j$ relative to $1, \ldots, s-1$. Now the only $(n-k)$-minors that are not in $R_{s}$ are the ones in which the relative order of $s-k$ and $j-k$ is determined by the relative order of $s$ and $j$ in $p$. Since we know the exact value of $x_{s-k, j-k}-y_{s-k, j-k}$, we can determine the relative order of $s$ and $j$ in $p$ : namely $s<_{p} j$ if and only if $x_{s-k, j-k}-y_{s-k, j-k}>0$.

Now we have determined the position of $j$ relative to $1,2, \ldots, j-1$ for all $k+2 \leq$ $j \leq n$. Therefore we know the relative order of all entries of $p$, which means that we have reconstructed $p$, and the result follows.

Example 2.7. Consider $M_{2}(p)=\left\{13245,14235^{2}, 14325,15324,23415^{2}, 24315^{4}, 24351\right.$, $\left.25341^{2}, 25431,32415,42135,42315^{2}, 43215,53241\right\}$. We will reconstruct $p$ from $M_{2}(p)$ following the algorithm in the proof of Theorem 2.6.

1. We determine $x_{1,2}=5$ and $x_{2,1}=16$. Therefore $2>_{p} 1$.

2. Let $i_{3}$ be the number of entries of $\{1,2\}$ that appear before 3 in $p$. Using Formula 2.3 we have

$$
\frac{5}{\left(\begin{array}{c}
7-3 \\
2-3+2
\end{array}\right)}-\frac{\left(\begin{array}{c}
7-3+1 \\
2-3+1
\end{array}\right)+(3-1)\left(\begin{array}{c}
7-3 \\
2-3+1
\end{array}\right)}{\left(\begin{array}{c}
7-3 \\
2-3+2
\end{array}\right)} \leq i_{3} \leq \frac{5}{\left(\begin{array}{c}
7-3 \\
2-3+2
\end{array}\right)} .
$$

Therefore $i_{3}=1$ and $2<_{p} 3<_{p} 1$.

3. To determine the position of 4 relative to $1,2,3$ we consider the relative positions of 1 and 2 in the 2-minors of $p$. We have four 2-minors $q$ obtained when 1 is deleted and 2 and 3 are not deleted that have $1<_{q} 2$. Therefore we have one 2-minor $r$ in which 4 acts as 2 and $1<_{r} 2$, which is the case only when $2<_{p} 4<_{p} 3<_{p} 1$. 
4. To determine the position of 5 relative to $1,2,3$ we consider the relative positions of 1 and 3 in the 2 -minors of $p$. We have six 2 -minors $q$ with $1<_{q} 3$. There are three 2 -minors in which 1 precedes 3 and their relative order is determined by the relative order of 1,2,3 and 4 in $p$. Therefore there are three 2-minors with 1 preceding 3 in which the entry 5 from $p$ acts as 3 . This happens if and only if $2<_{p} 3<_{p} 5<_{p} 1$. In the general case in order to determine the relative order of 4 and 5 , we will need to consider the relative positions of 2 and 3 in the 2-minors. But here we already have $4<_{p} 3<_{p} 5$ and we can conclude that $2<_{p} 4<_{p} 3<_{p} 5<_{p} 1$.

5. Similarly we can determine that $2<_{p} 6<_{4}<_{p} 3<_{p} 5<_{p} 1$ and that $2<_{p} 6<_{4}<_{p}$ $3<_{p} 5<_{p} 1<_{p} 7$.

Therefore $p=2643517$.

\section{Upper Bound on $N_{k}$}

In this section we find an upper bound for $N_{k}$. By Theorem $2.6 N_{k} \leq \max _{i}\left\{\left\lfloor b_{i}+1\right\rfloor\right\}$ where $b_{i}$ is the greater root of the equation $n^{2}-n[2 k+1+i(k-i+2)]+(k+1)[k+(i-1)(k-i+2)]=0$ for all $2 \leq i \leq k+1$.

Lemma 3.1. Let $k$ be a positive integer. If $k=2 m$ for some positive integer $m$, then $N_{k} \leq\left\lfloor b_{m}^{\prime}+1\right\rfloor$ where $b_{m}^{\prime}$ is the greater root of $n^{2}-n\left(m^{2}+6 m+2\right)+(2 m+1)\left(m^{2}+3 m\right)=0$. If $k=2 m-1$ for some positive integer $m$, then $N_{k} \leq\left\lfloor b_{m}^{\prime \prime}+1\right\rfloor$ where $b_{m}^{\prime \prime}$ is the greater root of $n^{2}-n\left(m^{2}+5 m-1\right)+2 m\left(m^{2}+2 m-2\right)=0$.

Proof. One can check that $\max _{i}\left\{b_{i}\right\}=b_{m+1}$ when $k=2 m$ and $\max _{i}\left\{b_{i}\right\}=b_{m}$ when $k=2 m-1$. Thus we obtain the corresponding inequalities.

Theorem 3.2. $N_{k}<\frac{k^{2}}{4}+2 k+4$.

Proof. Let $x_{k}=\frac{k^{2}}{4}+2 k+3$ and $f_{i}(x)=x^{2}-x[2 k+1+i(k-i+2)]+(k+1)[k+(i-1)(k-i+2)]$. To show that $x_{k}$ is larger than the largest root of each $f_{i}$, we will check that $f_{i}\left(x_{k}\right)>0$ and $x_{k}$ is larger than the $x$-value where $f_{i}$ achieves its minimum. By taking the derivative of $f_{i}$, we can see the second condition holds because

$$
\frac{2 k+1+i(k-i+2)}{2} \leq \frac{2 k+1+\frac{(k+2)^{2}}{4}}{2}=\frac{k^{2}}{8}+\frac{3 k}{2}+1<\frac{k^{2}}{4}+2 k+3 .
$$

For the first, observe that

$$
f_{i}\left(x_{k}\right)=\frac{k^{2}}{16}(k-2 i+2)^{2}+\frac{k}{4}(k-2 i+1)^{2}+\frac{1}{2}\left(k-2 i+\frac{3}{2}\right)^{2}+\frac{k}{4}+\frac{23}{8}>0 .
$$

Now it follows that $N_{k}<\left\lfloor x_{k}+1\right\rfloor<\frac{k^{2}}{4}+2 k+4$.

Using Lemma 3.1 we can easily compute $N_{1}$ and $N_{2}$, simplifying on the argument of Smith [4]. 
Lemma 3.3. $N_{1}=5$.

Proof. From Lemma 3.1 we have that $N_{1} \leq 5$. Since $M_{1}(2413)=M_{1}(3142)=$ $\left\{132^{1}, 213^{1}, 231^{1}, 312^{1}\right\}$, we have $N_{1}=5$.

Lemma 3.4. $N_{2}=6$.

Proof. From Lemma 3.1 we have that $N_{2} \leq 8$. With a computer check we verified that $M_{2}(p)$ uniquely determines $p$ when $p$ is of length 6 and 7 and we also have $M_{2}(25134)=$ $M_{2}(41253)=\left\{123^{2}, 132^{2}, 213^{2}, 231^{1}, 312^{3}\right\}$. Therefore $N_{2}=6$.

\section{Lower Bound on $N_{k}$}

Now that we have an upper bound for $N_{k}$ we proceed with finding a lower bound that disproves the conjecture of Smith [3] that $N_{k}=k+4$. Since the multiset $\cup_{q \in M_{k-1}(p)} M_{1}(q)$ contains $n-k+1$ copies of each of the minors in $M_{k}(p)$, the following lemma is clear.

Lemma 4.1 ([4], Lemma 5.1). Let $p$ and $q$ be permutations of length $n$ such that $M_{k-1}(p)=M_{k-1}(q)$, then $M_{k}(p)=M_{k}(q)$

Notation 4.2. Let $p_{1}$ and $p_{2}$ be permutations of length $n_{1}$ and $n_{2}$. We define $q=p_{1} \oplus p_{2}$ to be the permutation of length $n_{1}+n_{2}$ such that $q(i)=p_{1}(i)$ for $1 \leq i \leq n_{1}$ and $q(i)=n_{1}+p_{2}\left(i-n_{1}\right)$ for $n_{1}+1 \leq i \leq n_{1}+n_{2}$.

The next proposition will be useful for determining a lower bound on $N_{k}$. It will imply that if $N_{k}>n$, then $N_{k+m-n}>m$ where $m>n$.

Proposition 4.3. If there are permutations $p_{1} \neq p_{2}$ of length $n$ such that $M_{k}\left(p_{1}\right)=$ $M_{k}\left(p_{2}\right)$, then for any integer $m>n$ there exist permutations $q_{1} \neq q_{2}$ of length $m$ such that $M_{k+m-n}\left(q_{1}\right)=M_{k+m-n}\left(q_{2}\right)$.

Proof. The proof of the proposition is an extension of the proof of Proposition 5.3 in [4].

Let $p_{1} \neq p_{2}$ be permutations of length $n$ such that $M_{k}\left(p_{1}\right)=M_{k}\left(p_{2}\right)$. Let $q_{1}=p_{1} \oplus$ $12 \ldots(m-n)$ and $q_{2}=p_{2} \oplus 12 \ldots(m-n)$. Therefore $q_{1} \neq q_{2}$. Let $r_{1} \in M_{k+m-n}\left(q_{1}\right)$ and $r_{2} \in M_{k+m-n}\left(q_{2}\right)$. Then they must be in the form $r_{1}=p_{1}^{\prime} r_{1}^{\prime}$ and $r_{2}=p_{2}^{\prime} r_{2}^{\prime}$ where

$$
\begin{aligned}
& p_{1}^{\prime} \in M_{n_{1}}\left(p_{1}\right) \text { for some } k \leq n_{1} \leq \min \{n, k+m-n\} \text { and } r_{1}^{\prime}=\left(n-n_{1}+1\right) \ldots(n-k) \\
& p_{2}^{\prime} \in M_{n_{2}}\left(p_{2}\right) \text { for some } k \leq n_{2} \leq \min \{n, k+m-n\} \text { and } r_{2}^{\prime}=\left(n-n_{2}+1\right) \ldots(n-k) .
\end{aligned}
$$

Since $M_{k}\left(p_{1}\right)=M_{k}\left(p_{2}\right)$, from Lemma 4.1 it follows that $M_{l}\left(p_{1}\right)=M_{l}\left(p_{2}\right)$ for all integers $k \leq$ $l \leq n$. Therefore there exist bijections $f_{l}: M_{l}\left(p_{1}\right) \rightarrow M_{l}\left(p_{2}\right)$. We define $f: M_{k+m-n}\left(q_{1}\right) \rightarrow$ $M_{k+m-n}\left(q_{2}\right)$ as follows: $f\left(p_{1}^{\prime} r_{1}^{\prime}\right)=f_{n_{1}}\left(p_{1}^{\prime}\right) \oplus r_{1}^{\prime}$. Since $f_{n_{1}}$ is a bijection we have that $f$ is a bijection and it follows that $M_{k+m-n}\left(q_{1}\right)=M_{k+m-n}\left(q_{2}\right)$.

Corollary 4.4. If $N_{k}>n$, then $N_{k+m}>n+m$. 
In [4] Smith conjectures that $N_{k}=k+4$. A counterexample to this conjecture is $M_{4}(68573142)=M_{4}(75862413)=\left\{2413^{1}, 2431^{4}, 3142^{1}, 3241^{4}, 3412^{9}, 3421^{13}, 4132^{4}\right.$, $\left.4213^{4}, 4231^{8}, 4312^{13}, 4321^{9}\right\}$. The following proposition gives a lower bound for $N_{k}$.

Theorem 4.5. $N_{k}>k+\log _{2} k$.

Proof. Let $p_{1}$ and $p_{2}$ be two distinct permutations of length $k+m$ such that $M_{k}\left(p_{1}\right)=$ $M_{k}\left(p_{2}\right)$. We will show how to construct permutations $q_{1}$ and $q_{2}$ of length $2 m+2 k$ such that $M_{m+2 k-1}\left(q_{1}\right)=M_{m+2 k-1}\left(q_{2}\right)$. Let $q_{1}=p_{1} \oplus p_{2}$ and $q_{2}=p_{2} \oplus p_{1}$.

We consider $M_{m+2 k-1}(q)$ as the union of the following three sets:

$$
\begin{gathered}
R_{1}(q)=\left\{r \in M_{m+2 k-1}(q) \mid r \text { is obtained when the first } m+k\right. \text { entries are among } \\
\text { the deleted entries }\} \\
R_{2}(q)=\left\{r \in M_{m+2 k-1}(q) \mid r \text { is obtained when the last } m+k\right. \text { entries are among } \\
\text { the deleted entries }\} \\
R_{3}(q)=\left\{r \in M_{m+2 k-1}(q) \mid r \notin R_{1}\left(q_{1}\right), r \notin R_{2}\left(q_{2}\right)\right\} .
\end{gathered}
$$

We have $M_{m+2 k-1}(q)=R_{1}(q) \cup R_{2}(q) \cup R_{3}(q)$ and $\left|M_{m+2 k-1}(q)\right|=\left|R_{1}(q)\right|+\left|R_{2}(q)\right|+\left|R_{3}(q)\right|$.

We observe that $R_{1}\left(q_{1}\right)=R_{2}\left(q_{2}\right)$ and $R_{2}\left(q_{1}\right)=R_{1}\left(q_{2}\right)$. We will show that $R_{3}\left(q_{1}\right)=$ $R_{3}\left(q_{2}\right)$. We have that for each $r^{\prime} \in R_{3}\left(q_{1}\right), r^{\prime}=r_{1} \oplus r_{2}$ where $r_{1} \in M_{m_{1}}\left(p_{1}\right)$ and $r_{2} \in M_{m_{2}}\left(p_{2}\right)$ and $k \leq m_{1}, m_{2}<m+k$. Since $M_{k}\left(p_{1}\right)=M_{k}\left(p_{2}\right)$ we know from Lemma 4.1 that $M_{s}\left(p_{1}\right)=$ $M_{s}\left(p_{2}\right)$ when $s \geq k$. Therefore there are bijections $f_{i}: M_{m_{i}}\left(p_{1}\right) \rightarrow M_{m_{i}}\left(p_{2}\right)$ and we can define the bijection $f: R_{3}\left(q_{1}\right) \rightarrow R_{3}\left(q_{2}\right)$ as follows: $f\left(r^{\prime}\right)=f\left(r_{1} \oplus r_{2}\right)=f_{1}\left(r_{1}\right) \oplus f_{2}\left(r_{2}\right)$. Hence $R_{3}\left(q_{1}\right)=R_{3}\left(q_{2}\right)$ and $M_{m+2 k-1}\left(q_{1}\right)=M_{m+2 k-1}\left(q_{2}\right)$.

Since $N_{1}=5$ there are two permutations $p_{1} \neq p_{2}$ of length 4 such that $M_{1}\left(p_{1}\right)=$ $M_{1}\left(p_{2}\right)$. Now for every fixed positive integer $m, 2^{m-1}-m$ has the property that there are two permutations $q_{1} \neq q_{2}$ of length $2^{m-1}$ such that $M_{2^{m-1}-m}\left(q_{1}\right)=M_{2^{m-1}-m}\left(q_{2}\right)$. From Proposition 4.3 it follows that for any $k \geq 2^{m-1}-m$ there are two permutations $q_{1} \neq q_{2}$ of length $k+m$ such that $M_{k}\left(q_{1}\right)=M_{k}\left(q_{2}\right)$. Therefore $N_{k}>k+m$ for all $k>2^{m-1}-m$.

Let $k^{\prime}=2^{m-1}+c$ where $0 \leq c<2^{m-1}$. According to Corollary 4.4 we have

$$
N_{k^{\prime}} \geq N_{2^{m-1}-m}+m+c>2^{m-1}+m+c>k^{\prime}+\log _{2} k^{\prime}
$$

It follows that $N_{k}>k+\log _{2} k$.

\section{Reconstruction from Sets}

In this section we will focus our attention on the sets of minors of permutations and when they give enough information for the reconstruction of the starting permutation. We will show that if permutations of length $n$ are reconstructible from their sets of $(n-k)$-minors, then permutations of length $m>n$ are reconstructible from their sets of $(m-k)$-minors.

Definition 5.1. Let $M^{\prime}(p)$ be the set underlying the multiset $M(p)$. 
Definition 5.2. Let $N_{k}^{\prime}$ be the smallest integer such that for all permutations $p$ and $q$ of length $n \geq N_{k}, M_{k}^{\prime}(p)=M_{k}^{\prime}(q)$ implies $p=q$. If no such integer exists we will write $N_{k}^{\prime}=\infty$.

Similarly to $N_{k}$, if $N_{k}^{\prime}$ exists for some $k$, then distinct permutations of length $n \geq N_{k}^{\prime}$ have distinct sets of $(n-k)$-minors and there exist distinct permutations $q$ and $r$ of length $N_{k}^{\prime}-1$ such that $M_{k}^{\prime}(q)=M_{k}^{\prime}(r)$.

Notation 5.3. Let $p$ be a permutation. We denote the inverse permutation of $p$ by $i n v(p)$ and we denote the reverse permutation of $p$ by $\operatorname{rev}(p)$.

Lemma 5.4. Let $p$ be a fixed permutation. The following statements are equivalent:

1. $M_{k}^{\prime}(p)$ uniquely determines $p$,

2. $M_{k}^{\prime}(\operatorname{rev}(p))$ uniquely determines $p$,

3. $M_{k}^{\prime}(\operatorname{inv}(p))$ uniquely determines $p$.

Proof. The lemma follows from the facts that

$$
\begin{aligned}
& M_{k}^{\prime}(\operatorname{rev}(p))=\left\{\operatorname{rev}(q) \mid q \in M_{k}^{\prime}(p)\right\} \\
& M_{k}^{\prime}(\operatorname{inv}(p))=\left\{\operatorname{inv}(q) \mid q \in M_{k}^{\prime}(p)\right\}
\end{aligned}
$$

Notation 5.5. Let $q$ be a permutation of length $n$. Then $p=q \backslash\{i\}$ denotes the permutation obtained from $q$ by deleting the entry $i$.

Notation 5.6. Let $q$ be a permutation of length $n$. Let $r \in M_{k}^{\prime}(p)$ be obtained from $q$ when the entry $i$ was deleted. Then $p=r+{ }_{q}\{i\}$ denotes the $(n-k+1)$-minor of $q$ such that $r=p \backslash\left\{i_{1}\right\}$ where the entry $i$ from $q$ acts as $i_{1}$ in $p$.

Lemma 5.7. Suppose $M_{k}^{\prime}\left(p_{1}\right)=M_{k}^{\prime}\left(p_{2}\right)$ implies $p_{1}=p_{2}$ for all permutations $p_{1}, p_{2}$ of length $n$. Let $q_{1}$ and $q_{2}$ be permutations of length $n+1$ such that $q_{1} \backslash\{n+1\} \neq q_{2} \backslash\{n+1\}$. Then $M_{k}^{\prime}\left(q_{1}\right)=M_{k}^{\prime}\left(q_{2}\right)$ implies that $q_{1}=q_{2}$.

Proof. Assume that $M_{k}^{\prime}\left(q_{1}\right)=M_{k}^{\prime}\left(q_{2}\right)$. Let $p_{1}=q_{1} \backslash\{n+1\}$ and $p_{2}=q_{2} \backslash\{n+1\}$. Since $p_{1} \neq p_{2}$, and $p_{1}$ and $p_{2}$ are permutations of length $n$, it follows that $M_{k}^{\prime}\left(p_{1}\right) \neq M_{k}^{\prime}\left(p_{2}\right)$. So there is a permutation $r$ such that $r \in M_{k}^{\prime}\left(p_{1}\right)$ and $r \notin M_{k}^{\prime}\left(p_{2}\right)$. Therefore $r \in M_{k+1}^{\prime}\left(q_{1}\right)$. Now consider $r^{\prime}=r+{ }_{q_{1}}\{n+1\}$. We have that $r^{\prime} \in M_{k}^{\prime}\left(q_{1}\right)$ and since we have assumed that $M_{k}^{\prime}\left(q_{1}\right)=M_{k}^{\prime}\left(q_{2}\right)$, it follows that $r^{\prime} \in M_{k}^{\prime}\left(q_{2}\right)$. If $r^{\prime}$ was obtained as an $(n+1-k)$-minor of $q_{2}$ by deleting the entry $n+1$, then $r^{\prime} \in M_{k-1}^{\prime}\left(p_{2}\right)$. Hence $r \in M_{k}^{\prime}\left(p_{2}\right)$, which contradicts our assumption. Therefore we assume that $r^{\prime}$ was obtained from $q_{2}$ without deleting the entry $n+1$. Thus the entry $n+1$ in $q_{2}$ will be the entry $n+1-k$ in $r^{\prime}$, which means that $r^{\prime} \backslash\{n+1-k\} \in M_{k}^{\prime}\left(p_{2}\right)$. At the same time $r=r^{\prime} \backslash\{n+1-k\}$ since the entry $n+1$ in $q_{1}$ is the entry $n+1-k$ in $r^{\prime}$. Now we have again that $r \in M_{k}^{\prime}\left(p_{2}\right)$, which contradicts our assumption. 
Theorem 5.8. If $M_{k}^{\prime}\left(p_{1}\right)=M_{k}^{\prime}\left(p_{2}\right)$ implies that $p_{1}=p_{2}$ for any two permutations $p_{1}$ and $p_{2}$ of length $n>1$, then $M_{k}^{\prime}\left(q_{1}\right)=M_{k}^{\prime}\left(q_{2}\right)$ implies $q_{1}=q_{2}$ for any two permutations $q_{1}$ and $q_{2}$ of length $m>n$.

Proof. We will show that if $M_{k}^{\prime}(p)$ uniquely determines every permutation $p$ of length $n$, then $M_{k}^{\prime}(q)$ uniquely determines every permutation $q$ of length $n+1$.

Assume that for any two permutations $p_{1} \neq p_{2}$ of length $n M_{k}^{\prime}\left(p_{1}\right) \neq M_{k}^{\prime}\left(p_{2}\right)$. Suppose that there exist permutations $q_{1} \neq q_{2}$ of length $n+1$ such that $M_{k}^{\prime}\left(q_{1}\right)=M_{k}^{\prime}\left(q_{2}\right)$. If $q_{1} \backslash\{n+1\} \neq q_{2} \backslash\{n+1\}$, then from Lemma 5.7 it follows that $q_{1}=q_{2}$. Also if $i n v\left(q_{1}\right) \backslash\{n+$ $1\} \neq \operatorname{inv}\left(q_{2}\right) \backslash\{n+1\}$, then we will have that $M_{k}^{\prime}\left(i n v\left(q_{1}\right)\right)=M_{k}^{\prime}\left(i n v\left(q_{2}\right)\right)$ implies $i n v\left(q_{1}\right)=$ $\operatorname{inv}\left(q_{2}\right)$. From Lemma 5.4 we conclude that $M_{k}^{\prime}\left(q_{1}\right)=M_{k}^{\prime}\left(q_{2}\right)$ implies that $q_{1}=q_{2}$. Similarly if $\operatorname{rev}\left(\operatorname{inv}\left(q_{1}\right)\right) \backslash\{n+1\} \neq \operatorname{rev}\left(\operatorname{inv}\left(q_{2}\right)\right) \backslash\{n+1\}$, then $M_{k}^{\prime}\left(q_{1}\right)=M_{k}^{\prime}\left(q_{2}\right)$ implies that $q_{1}=q_{2}$.

Now assume that $q_{1} \backslash\{n+1\}=q_{2} \backslash\{n+1\}$, inv $\left(q_{1}\right) \backslash\{n+1\}=\operatorname{inv}\left(q_{2}\right) \backslash\{n+1\}$ and $\operatorname{rev}\left(\operatorname{inv}\left(q_{1}\right)\right) \backslash\{n+1\}=\operatorname{rev}\left(\operatorname{inv}\left(q_{2}\right)\right) \backslash\{n+1\}$. Suppose $q_{1} \neq q_{2}$. From $q_{1} \backslash\{n+1\}=q_{2} \backslash\{n+1\}$ we must have that $n+1$ is at different positions in $q_{1}$ and $q_{2}$. The equality $\operatorname{inv}\left(q_{1}\right) \backslash\{n+1\}=$ $\operatorname{inv}\left(q_{2}\right) \backslash\{n+1\}$ means that $n+1$ is at position $n+1$ in one of $q_{1}$ and $q_{2}$. Then the equality $\operatorname{rev}\left(\operatorname{inv}\left(q_{1}\right)\right) \backslash\{n+1\}=\operatorname{rev}\left(\operatorname{inv}\left(q_{2}\right)\right) \backslash\{n+1\}$ means that $n+1$ is in position 1 in one of $q_{1}$ and $q_{2}$. Suppose $q_{1}(1)=n+1$ and $q_{2}(n+1)=n+1$. Since $\operatorname{inv}\left(q_{1}\right) \backslash\{n+1\}=i n v\left(q_{2}\right) \backslash\{n+1\}$ we must have that $\left(q_{2} \backslash\{n+1\}\right)(1)=n$. Since $\operatorname{rev}\left(\operatorname{inv}\left(q_{1}\right)\right) \backslash\{n+1\}=\operatorname{rev}\left(\operatorname{inv}\left(q_{2}\right)\right) \backslash\{n+1\}$, we must have that $\left(q_{1} \backslash\{n+1\}\right)(n)=n$. But this is impossible because $q_{1} \backslash\{n+1\}=q_{2} \backslash\{n+1\}$ and $n>1$.

It follows that $M_{k}^{\prime}\left(q_{1}\right)=M_{k}^{\prime}\left(q_{2}\right)$ implies that $q_{1}=q_{2}$.

We state the following corollary, which we will apply to the problem of reconstruction from multisets to find the exact value of $N_{3}$.

Corollary 5.9. Let $q_{1}$ and $q_{2}$ be permutations of length $n+1$. If any of the inequalities holds: $M_{k}^{\prime}\left(q_{1} \backslash\{n+1\}\right) \neq M_{k}^{\prime}\left(q_{2} \backslash\{n+1\}\right), M_{k}^{\prime}\left(\operatorname{rev}\left(q_{1}\right) \backslash\{n+1\}\right) \neq M_{k}^{\prime}\left(\operatorname{rev}\left(q_{2}\right) \backslash\{n+\right.$ $1\}), M_{k}^{\prime}\left(\operatorname{inv}\left(q_{1}\right) \backslash\{n+1\}\right) \neq M_{k}^{\prime}\left(\operatorname{inv}\left(q_{2}\right) \backslash\{n+1\}\right)$, or $M_{k}^{\prime}\left(\operatorname{rev}\left(\operatorname{inv}\left(q_{1}\right)\right) \backslash\{n+1\}\right) \neq$ $M_{k}^{\prime}\left(\operatorname{rev}\left(\operatorname{inv}\left(q_{2}\right)\right) \backslash\{n+1\}\right)$, then $M_{k}^{\prime}\left(q_{1}\right) \neq M_{k}^{\prime}\left(q_{2}\right)$ and $M_{k}\left(q_{1}\right) \neq M_{k}\left(q_{2}\right)$.

Proof. This follows from the proof of Theorem 5.8.

In her paper [3] Smith shows that $N_{3}=7,10,11,12$, or 13 . Now we find the exact value of $N_{3}$.

Proposition 5.10. $N_{3}=7$

Proof. From Lemma 3.1 we have that $N_{3} \leq 11$. Using a computer check one can see that $M_{3}(p)$ uniquely determines $p$ when $p$ has length 7,8 and 9 . Suppose that there exist two permutations $q_{1}$ and $q_{2}$ of length 10 such that $M_{3}\left(q_{1}\right)=$ $M_{3}\left(q_{2}\right)$. According to Corollary 5.9 we should have that $M_{3}^{\prime}\left(q_{1} \backslash\{10\}\right)=M_{3}^{\prime}\left(q_{2} \backslash\{10\}\right)$, $M_{3}^{\prime}\left(\operatorname{rev}\left(q_{1}\right) \backslash\{10\}\right)=M_{3}^{\prime}\left(\operatorname{rev}\left(q_{2}\right) \backslash\{10\}\right), \quad M_{3}^{\prime}\left(\operatorname{inv}\left(q_{1}\right) \backslash\{10\}\right)=M_{3}^{\prime}\left(\operatorname{inv}\left(q_{2}\right) \backslash\{10\}\right)$ and $M_{3}^{\prime}\left(\operatorname{rev}\left(\operatorname{inv}\left(q_{1}\right)\right) \backslash\{10\}\right)=M_{3}^{\prime}\left(\operatorname{rev}\left(\operatorname{inv}\left(q_{2}\right)\right) \backslash\{10\}\right)$. With a computer search we find that the only permutations of length 9 that have the same sets of (6)-minors 
are $M_{3}^{\prime}(132465798)=M_{3}^{\prime}(132465879)=M_{3}^{\prime}(132546879)=M_{3}^{\prime}(213546879)$ and $M_{3}^{\prime}(897564231)=M_{3}^{\prime}(978564231)=M_{3}^{\prime}(978645231)=M_{3}^{\prime}(978645312)$. We observe that there is no way to choose all $\left.q_{i} \backslash\{10\}, \operatorname{rev}\left(q_{i}\right) \backslash\{10\}, \operatorname{inv}\left(q_{i}\right) \backslash\{10\}\right), \operatorname{rev}\left(\operatorname{inv}\left(q_{i}\right)\right) \backslash\{10\}$ for $i=1,2$ among these permutations. Therefore $M_{3}(p)$ uniquely determines $p$ when $p$ has length 10. Finally we have $M_{3}(623514)=M_{3}(631452)=\left\{123^{2}, 132^{3}, 213^{2}, 231^{3}\right.$, $\left.312^{7}, 321^{4}\right\}$. It follows that $N_{3}=7$.

The following proposition shows similarities between the sets and multisets of minors.

Proposition 5.11. If there are permutations $p_{1} \neq p_{2}$ of length $n$ such that $M_{k}^{\prime}\left(p_{1}\right)=$ $M_{k}^{\prime}\left(p_{2}\right)$, then for any integer $m>n$ there exist permutations $q_{1} \neq q_{2}$ of length $m$ such that $M_{k+m-n}^{\prime}\left(q_{1}\right)=M_{k+m-n}^{\prime}\left(q_{2}\right)$.

Proof. The proof is analogous to the proof of Proposition 4.3.

Proposition 5.12. $N_{k}^{\prime}>2 k$.

Proof. When $k=1$ the proposition is true. Let $k>1$. Consider the permutations $p=$ $12 \ldots(k-1)(k+1) k(k+2)(k+3) \ldots(2 k)$ and $q=12 \ldots(k-1) k(k+2)(k+1)(k+3) \ldots(2 k)$. We observe that $M_{k}^{\prime}(p)=M_{k}^{\prime}(q)=\left\{1 \ldots k, r_{1}, \ldots, r_{k-1}\right\}$ where $r_{i}$ is obtained from the permutation $12 \ldots k$ by transposing $i$ and $i+1$. Thus $N_{k}^{\prime}>2 k$ for all positive integers $k$.

Note: Using a computer check and Theorem 5.8 we find that $N_{1}^{\prime}=5$, which has also been proved by Ginsburg [1] and Smith [4], $N_{2}^{\prime}=7$, and $N_{3}^{\prime}>9$.

\section{Reconstruction of Layered Permutations}

In Section 2 we proved that $N_{k}<\infty$ for all $k$, but we still do not have that $N_{k}^{\prime}<\infty$ for all $k$. One approach to proving this will be to divide permutations into classes that are recognizable from their multisets or sets of minors and prove the existence of $N_{k}^{\prime}$ for those classes. Layered permutation are one example. The following theorem suggests that we can analyze such classes on their own.

Theorem 6.1. Let $S$ be a set of permutations of length smaller than c. Let $P=\{p \mid p$ is of length $l \geq c$ and $r_{i}$ is not a minor of $p$ for all $\left.r_{i} \in S\right\}$. If $M_{k}^{\prime}\left(p_{1}\right) \neq M_{k}^{\prime}\left(p_{2}\right)$ for any two different permutations $p_{1}, p_{2} \in P$ of length $n$, then $M_{k}^{\prime}\left(q_{1}\right) \neq M_{k}^{\prime}\left(q_{2}\right)$ for any two different permutations $q_{1}, q_{2} \in P$ of length $m>n$.

Proof. The proof is analogous to the proof of Theorem 5.8 taking into account the fact that if we have a permutation $q$ of length $m$ and $q \in P$, then $p \in P, \operatorname{inv}(p) \in P$ and $\operatorname{rev}(p) \in P$ for all $p \in M_{1}(q)$.

Definition 6.2. Let $p$ be a permutation of length $n$. We say that $p$ is a layered permutation if there exists a division $p=q_{1} \cdots q_{i}$ such that $q_{j}$ is a decreasing sequence of $m_{j}$ consecutive integers for all $1 \leq j \leq i$, and every entry in $q_{j_{1}}$ is smaller than every entry in $q_{j_{2}}$ when $j_{1}<j_{2}$. We call $q_{1}, \ldots, q_{i}$ blocks of the permutation $p$. 
Note: Consider the type of permutations that can be written in the form $p=q_{1} \ldots q_{i}$ such that $q_{j}$ is an increasing sequence of $m_{j}$ consecutive integers, and every entry in $q_{j_{1}}$ is greater than every entry in $q_{j_{2}}$ when $j_{1}<j_{2}$. There is a bijection between the layered permutations and this class of permutations since the permutations in both of them are uniquely determined by the sequence $m_{1}, m_{2}, \ldots, m_{i}$. Therefore the following results that we will prove for layered permutations will hold for both classes of permutations.

Example 6.3. Consider the layered permutation 321765498 and the corresponding permutation 789345612 with increasing subsequences. They have the same determining sequence $3,4,2$.

Lemma 6.4. Let $n-k \geq 3$. A permutation $p$ of length $n$ is layered if and only if $M_{k}(p)$ contains only layered permutations.

Proof. Layered permutations are permutations that do not have 231 and 312 as (3)minors. If $m \geq k$, a permutation does not contain a $(k)$-minor if and only if none of its $(m)$-minors contains that $(k)$-minor, and the lemma follows.

Definition 6.5. Let $L_{k}^{\prime}$ be the smallest integer such that for all layered permutations $p$ and $q$ of length $n \geq L_{k}^{\prime}, M_{k}^{\prime}(p)=M_{k}^{\prime}(q)$ implies $p=q$. If no such integer exists we will write $L_{k}^{\prime}=\infty$.

We are interested in the number $L_{k}^{\prime}$ since according to Lemma 6.4 we can determine whether $p$ is a layered permutation from the set $M_{k}^{\prime}(p)$ when $n-k \geq 3$. We also know $L_{k}^{\prime} \leq N_{k}^{\prime}$ since if distinct permutations of length $n$ have distinct sets of $(k)$-minors then distinct layered permutations of length $n$ have distinct sets of $(k)$-minors.

Proposition 6.6. $L_{k}^{\prime}>k+\log _{2} k$.

Proof. If $k=1$ the proposition is true. Now we consider the case when $k \geq 2$. Let $k=2^{m}$ for some positive integer $m$. There are $2^{k+\log _{2} k-1}$ layered permutations of length $k+\log _{2} k$ [2]. Also Lemma 6.4 gives us that there are $2^{\log _{2} k-1}=\frac{k}{2}$ possible different $\left(\log _{2} k\right)$-minors for layered permutations. Therefore the number of different sets that we can form using these $\left(\log _{2} k\right)$-minors is:

$$
\left(\begin{array}{l}
\frac{k}{2} \\
1
\end{array}\right)+\left(\begin{array}{l}
\frac{k}{2} \\
2
\end{array}\right)+\cdots+\left(\begin{array}{c}
\frac{k}{2} \\
\min \left\{\frac{k}{2},\left(\begin{array}{c}
k+\log _{2} k \\
k
\end{array}\right)\right\}
\end{array}\right) \leq 2^{\frac{k}{2}} .
$$

Since $2^{\frac{k}{2}}<2^{k+\log _{2} k-1}$ it follows that there will be two layered permutations of length $k+\log _{2} k$ that will have the same sets of $\left(\log _{2} k\right)$-minors. Hence $L_{k}^{\prime}>k+\log _{2} k$ when $k=2^{m}$.

Now let $k=2^{m}+c$ where $c<2^{m}$. Similarly to the proof of Theorem 4.5 we have

$$
L_{k}^{\prime} \geq L_{2^{m}}^{\prime}+c \geq 2^{m}+m+1+c>k+\log _{2} k .
$$

Thus $L_{k}^{\prime}>k+\log _{2} k$ for all positive integers $k$. 


\section{Open Questions}

This paper develops in three main directions - permutation reconstruction from multisets and sets of minors as well as permutation reconstruction from sets of minors within certain classes of permutations. In all three of them there are open questions.

1. Permutation reconstruction from multisets of minors:

Question 7.1. Can the bounds for $N_{k}$ be improved and is there an exact formula for $N_{k}$ ?

The next question was posed by Smith [4]

Question 7.2. Does the fact that distinct permutations of length $n$ have distinct multisets, imply that distinct permutations of length $m>n$ must have distinct multisets?

If the answer to Question 7.2 is positive, given the exact values of $N_{1}, N_{2}$ and $N_{3}$, this will suggest that probably the lower bound on $N_{k}$ is tighter and there will be room for improvement on the upper bound.

2. Permutation reconstruction from sets of minors:

Question 7.3. Does $N_{k}^{\prime}$ exist for all $k$ ?

A positive answer to the following question would prove the existence of $N_{k}^{\prime}$ for all $k$ :

Question 7.4. Does there exist a positive integer $m$ such that if $M_{k}^{\prime}\left(p_{1}\right)=M_{k}^{\prime}\left(p_{2}\right)$ implies $p_{1}=p_{2}$ for any permutations $p_{1}$ and $p_{2}$ of length $n$, then $M_{k+1}\left(q_{1}\right)=M_{k+1}\left(q_{2}\right)$ implies $q_{1}=q_{2}$ for any two permutations $q_{1}$ and $q_{2}$ of length $n+m$ ?

Also studying whether the difference $N_{k}^{\prime}-N_{k}$ is bounded, would give insight into how much information we lose about the starting permutation when we go from the multiset to the set of its minors.

3. Classes of permutations:

The work in all of the above questions can start by proving results for specific types of permutations. One example would be permutations that avoid certain minors similar to the layered permutations avoiding 231 and 312. Such classes of permutations can be identified from the multiset or the set of minors that we are considering. Therefore answering the questions about permutation reconstruction from multisets or sets of minors within a given class, would be a step in the direction of answering the same questions for all permutation. An example would be:

Question 7.5. Are sufficiently long layered permutations reconstructible from their sets of minors? 


\section{Acknowledgments}

This research was done at the University of Minnesota Duluth with the financial support of Bard College and University of Minnesota Duluth. I would like to thank Joseph Gallian, Philip Matchett and David Arthur for their help and encouragement. I would extend my thanks to Melanie Wood, Stephen Hartke and Reid Barton for their useful suggestions.

\section{References}

[1] John Ginsburg, Determining a permutation from its set of reductions, Ars Combinatoria, to appear

[2] R. Simion and F. W. Schmidt, Restricted permutations, European Journal of Combinatorics 6 (1985), 383-406.

[3] R. Smith, Combinatorial Algorithms Involving Pattern Containing and Avoiding Permutations, Ph.D. thesis, University of Florida, 2005

[4] R. Smith, Permutation reconstruction, Electronic Journal of Combinatorics (2006), $13(1)$

[5] S. M. Ulam, A Collection of Mathematical Problems, Wiley, New York, 1960. 